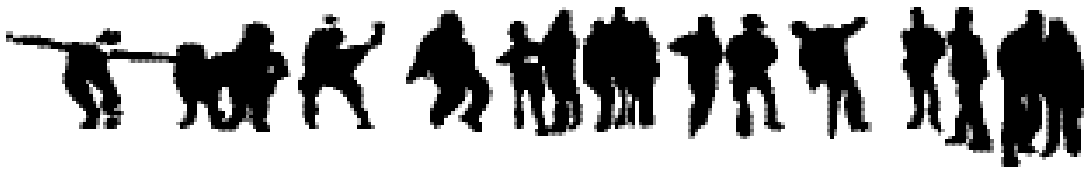

\section{Description de quelques caractéristiques communes aux opérations de dotations mas- sives en ordinateurs portables en France}

\author{
Mehdi Khaneboubi (EDA, Université Paris Descartes)
}

- RÉSUMÉ • Depuis la rentrée 2001, les élèves et les enseignants des collèges du département des Landes disposent d'un ordinateur portable. Les départements des Bouches-du-Rhône, d'Ille-et-Vilaine, de l'Oise et de la Corrèze ont aussi mis en œuvre dans les années 2000 des projets de dotation similaires. En plus des logiciels de bureautiques habituels les enseignants et les élèves ont la possibilité d'utiliser des répéticiels, des ENT ainsi que des services en ligne documentaires et parascolaires. Des entretiens et des observations de classes montrent que les enseignants rencontrés ont tendance à utiliser les logiciels reconnus comme légitimes dans leur champ disciplinaire. Leurs usages des TIC en classe restent dans la continuité de leurs utilisations des technologies antérieures.

- MOTS CLÉS • collège, ordinateur portable, enseignant

ABSTRACT • Since 2001, students and teachers in middle schools of a French south west area received a laptop. Since then, four others locals councils administration have implemented similar projects. In addition to the usual office software, teachers and students have the opportunity to use various online and outline software: educative software, learning platform, educative database... Interviews and observation show that teachers tend to use software recognized as legitimate in their subject matter. Their classroom use of is a continuation of their uses of previous technology.

- KEYWORDS • middle school, laptop, teachers

\section{Introduction}

Des projets d'équipement en ordinateurs portables ont été mis en œuvre par cinq conseils généraux français. Héritiers du plan Informatique pour tous au niveau départemental, ces déploiements constituent des actions politiques importantes dans le domaine des TICE. Le conseil général du département des Landes fut le premier à avoir effectué des prêts systématiques d'ordinateurs portables sur un tronçon de l'école obligatoire (landesinteractives.net, 2009). Lors de la rentrée 2002, tous les élèves et tous les professeurs de troisième de ce 
grand département rural peu peuplé, avaient à leur disposition un ordinateur portable et la possibilité de se connecter à internet dans les salles de classe de leur collège. Depuis, d'autres départements ont mis en œuvre des dispositifs similaires : le département des Bouches-du-Rhône en 2003 (" $\underline{\text { Ordina } 13}$ »), le département d'Ille-et-Vilaine en 2004 ( $\underline{\text { Ordi } 35}$ »), les départements de l'Oise (" Ordi 60 ») et de la Corrèze (« Ordicollège 19 ») en 2009 (Corrèze, 2009a, 2009b, 2009c). Notons que l'opération de l'Oise vise principalement à favoriser les utilisations de l'ordinateur à la maison. Dans ce département, les élèves ne peuvent pas amener les ordinateurs au collège.

L'objet de ce texte est de présenter, sur les opérations de dotations françaises, un point de vue personnel s'appuyant sur les éléments suivants :

- des observations de classes réalisées dans le département des Landes deux jours par semaine de novembre à avril en 2001-2002 et une journée par semaine de décembre à avril en 2004-2005 ;

- une cinquantaine d'entretiens informels conduits en 2009 dans 9 collèges de l'Oise alors que les enseignants et les élèves venaient d'être dotés ;

- deux questionnaires ont été distribués/dépouillés/analysés dans neuf collèges de l'Oise $(\mathrm{N}=200)$ et dans six collèges des Landes $(\mathrm{N}=144)$.

Bien que ces informations aient été collectées à des années d'intervalle, dans des lieux différents, les similarités dans les dispositifs, dans les discours des enseignants et dans les pratiques observées dans les Landes et déclarées dans l'Oise sont si grandes, qu'elles tendent à indiquer que les mécanismes et les processus en jeu dans les collèges des Landes et de l'Oise ont des points communs qui dépassent l'éloignement géographique et temporel. D'ailleurs, des articles de recherche portant sur les différents projets décrivent des discours et des pratiques convergentes (Brandt-Pomares et Boilevin, 2009 ; Daguet, 2007, 2009 ; Jaillet, 2004 ; Liautard, 2007 ; Rinaudo, 2008 ; Rinaudo et al., 2008). Enfin pour les projets les plus récents comme ceux de l'Oise et de la Corrèze, nous avons collecté des informations disponibles en ligne, principalement sur les supports de communication des conseils généraux.

Dans cette contribution, nous allons analyser les caractéristiques principales de ces opérations : matériel mis à disposition, environnements logiciels et modification éventuelle des enseignements. Nous avons ainsi cherché à présenter comment le matériel et les ressources sont utilisables et utilisés par les enseignants : quels instruments informatisés sont à disposition et dans quelle mesure ces instruments sont-ils susceptibles d'accompagner une évolution des pratiques pédagogiques dans les collèges? 


\section{Des politiques publiques voisines}

Parmi les points communs entre les dispositifs landais, isarien, breton et provençal on remarque les campagnes de communication qui escortent les dotations. Souvent, les conseils généraux ont présenté ces dispositifs comme une étape décisive pour réduire la fracture numérique (oise.fr, 2009), « favoriser de nouvelles pratiques pédagogiques » (Bellocq, 2004 p.2), « favoriser et développer les usages pédagogiques des technologies de l'information et de la communication pour l'éducation » (ordi35.org, 2009) , ou comme un moyen pour favoriser la réussite des élèves (Corrèze, 2009c).

L'accent est aussi mis sur leur caractère inédit et/ou massif. Ainsi, Ordina 13 fut présenté par le conseil général comme « une des plus importantes opérations TICE menée par une collectivité publique » (Liautard, 2007). De la même façon, le site $\operatorname{Ordi} 60$ indique qu'« une opération d'une telle ampleur est une première en France » et le site du projet landais mentionne qu'il s'agit d'une « opération de grande ampleur de modernisationi de l'équipement informatique de ses collèges ».

Lors de la première année du projet landais, les thèmes évoqués dans la campagne de communication du conseil général tournaient autour de l'idée qu'il s'agissait d'une action décisive pour l'avenir des élèves, pour celui du département sur le plan de la formation et de l'aménagement du territoire. L'argumentation laissait penser que l'économie du département serait catalysée par les ordinateurs portables dans les collèges. Par la suite, le dispositif fut présenté comme cherchant à " mettre en œuvre des actions qui aient des retombées sur l'ensemble des secteurs de l'activité humaine, mais aussi sur l'ensemble de son territoire " (Bellocq, 2004). Aujourd'hui, les objectifs présentés sur le site web du projet sont beaucoup plus modestes. Sur les sites des autres projets, ils sont souvent inexistants ou présentés de façon lapidaire. Mais il n'en reste pas moins que la communication faite autour des ordinateurs portables dans les collèges des Landes et des autres départements suggère qu'il s'agit d'actes politiques décisifs pour les départements en faveur de l'aménagement du territoire.

Bien que ces opérations soient coûteuses, elles ne marquent pas forcément une rupture avec les politiques précédentes à l'égard des établissements scolaires. Ainsi, bien souvent les chefs d'établissements de ces départements font remarquer que leurs demandes auprès des conseils généraux avaient bien plus de chances d'aboutir qu'auprès des rectorats. Aux dires des enseignants rencontrés, du point de vue des TICE, les départements des Landes et de l'Oise ont depuis les années 80 soutenu et doté les collèges d'ordinateurs, de serveurs, etc. 


\section{Une saturation technologique}

Initialement, les dotations visent à créer dans les collèges des environnements saturés par les technologies. Dans les Landes, en plus des imprimantes, des bornes Ethernet et des serveurs qui équipent les 35 collèges, 9000 ordinateurs, 900 vidéoprojecteurs et 800 tableaux interactifs sont dédiés aux usages pédagogiques des classes de quatrième et troisième (landesinteractives.net, 2010). En Ille-et-Vilaine lors de la rentrée 2004, 11000 machines ont été distribuées aux élèves de troisième dans tous les collèges du département (Rinaudo et al., 2008, p. 11). En 2007 dans les Bouches-du-Rhône le parc était composé de 60 000 portables (Liautard, 2007). Dans l'Oise en 2009, 21000 ordinateurs étaient à disposition des collégiens de cinquième et de quatrième (peo60.fr, 2010).

Suivant les départements, il s'agit d'un prêt ou d'un quasi-don. Dans les Landes, les élèves de quatrième reçoivent un ordinateur en début d'année qu'ils doivent restituer à la fin de leur année de troisième. En Ille-et-Vilaine, les élèves doivent rendre leurs ordinateurs à la fin de chaque année et pouvaient à la rentrée 2009/2010 acheter les machines mises en service en 2004. Dans l'Oise, les élèves peuvent garder l'ordinateur à la fin du collège en échange d'une caution peu élevée. Dans les Bouches-du-Rhône, il semblerait que la dotation soit soumise aux mêmes conditions que dans l'Oise.

Les trois opérations les plus anciennes ont été mises en œuvre dans une logique d'équipement des établissements et des salles de classe de façon à rendre possible une utilisation de ces technologies quelle que soit la discipline enseignée. Dans l'Oise, les élèves ne sont pas autorisés à amener leurs ordinateurs en classe ni même au collège. Même si les enseignants ont été dotés, les bâtiments n'ont pas été équipés en matériels périphériques supplémentaires et n’ont donc pas un taux d'élèves par ordinateur comparable aux autres projets. Cette caractéristique est probablement une façon pour la collectivité territoriale de ne pas s'immiscer davantage dans les enseignements et de ne pas avoir à câbler et aménager les salles de classe. En découle une différence importante entre les dotations : celles qui ciblent les collèges et la dotation isarienne qui vise plus particulièrement les élèves et leurs pratiques en dehors de l'école.

\section{Les solutions logicielles}

Les autorités territoriales fournissent généralement avec les machines, en plus des instruments bureautiques habituels, des encyclopédies, divers logiciels, des accès à des bases thématiques éducatives et des accès à des sites de soutien scolaire à distance. Les contenus à disposition sont davantage un complément à 
l'apprentissage. Les ressources documentaires offrent en outre la possibilité aux élèves un accès depuis leur foyer.

Dans les Landes, le projet initial visait à mettre en œuvre un cartable électronique qui devait remplacer les cartables ordinaires. Le conseil général avait envisagé d'utiliser exclusivement des livres numériques, mais la grande majorité des manuels fournis par les éditeurs étaient fort peu pratiques à utiliser (Daguet, 2009 ; Khaneboubi, 2007). Il s'agissait parfois d'un manuel papier scanné et transformé au format PDF avec des droits d'utilisations limités à l'année en cours. La lisibilité de ces documents était plutôt médiocre (Daguet, 2009).

Les instruments les plus utilisés en classe par les enseignants que j'ai observés dans les Landes et écoutés dans l'Oise, restent les traitements de texte, les tableurs et les logiciels de présentation. De façon plus large, ces enseignants utilisent avec les élèves des logiciels reconnus comme légitimes dans leur communauté (par exemple Audacity en musique, Gimp ou Paint Shop Pro en arts plastiques, GéoGebra en mathématiques, etc.). Les logiciels, du type Lirebel, Grambel, Math en poche, sont souvent utilisés lors des cours pour lesquels la pression évaluative est moindre. Ce sont le plus souvent des logiciels généralistes (suites bureautiques, lecteur vidéo, lecteur audio, messagerie instantanée, client $I R C$...) ou professionnels (des logiciels issus de Music lab, Photoshop, un SIG) qui sont utilisés dans les scénarios didactiques des enseignants. Les répéticiels restent l'apanage d'utilisations périphériques ou moins importantes (veilles de vacances, voyage scolaire d'une partie de la classe, fin de trimestre...).

Bien que Microsoft Windows soit le système d'exploitation le plus souvent choisi pour équiper les ordinateurs des élèves et des enseignants de même que Microsoft Windows-Serveur pour les serveurs de collèges, on note quelques exceptions à la domination de la firme de Redmond. Une des originalités du projet corrézien réside dans le fait qu'il s'agit de dotation de machines exclusivement sous Ubuntu. Dans les collèges de l'Oise une partie des serveurs tourne sous Apache. Enfin, Open Office s'est imposé sur la plupart des machines comme outil bureautique par défaut.

De façon générale, les logiciels libres sont assez présents sur les machines fournies par les départements. Dès 2003, Open Office a été installé sur les machines landaises. Microsoft avait pourtant lors de la première année du projet gascon, donné gratuitement Microsoft Office. Après l'ouverture du code de Star Office, le conseil général avait laissé la possibilité aux établissements d'acheter ou non une suite bureautique. Dans l'Oise, pour la première année du projet, Microsoft Office a aussi été donné gratuitement. 


\section{Quel renouveau dans les enseignements?}

Les enseignants dans les collèges qui ont globalement réussi à remettre en question les pédagogies traditionnelles sont plutôt ceux qui enseignent dans des disciplines historiquement utilisatrices de technologies comme la musique ou les arts plastiques. Ce résultat converge avec ce que présentent Larose, Grenon, Pearson, Marin, \& Lenoir, (2004). Dans un travail réalisé à l'école primaire, ils constatent que les enseignants considèrent souvent que les matières secondaires (sciences humaines et sciences de la nature au Québec) sont un espace dans lequel la maîtrise des contenus disciplinaires n'est pas essentielle. Ces disciplines, constituent davantage un espace où se développent des savoir-faire transversaux. Les auteurs estiment donc que l'usage des TIC en classe est lié au rapport aux savoirs des enseignants. On a constaté cette tendance pour les professeurs que nous avons rencontrés. Nous allons illustrer cette idée en présentant des extraits de journaux d'observations et d'entretiens tenus lors dès séjours dans des collèges. Ainsi, un enseignant isarien d'art plastique en ZEP décrit les réalisations de ses élèves dans sa discipline :

" Je leur fais faire des petites annonces un peu comme Les petites annonces d'Élie Seimoun. En quatrième, je leur demande de faire un flash info soit au format radio, soit au format vidéo, soit une affiche. En sixième, on fait de l'animation avec un appareil photo numérique, ils doivent raconter une histoire avec un objet. J'ai des élèves qui m'ont fait l'histoire d'un bout de bois qui mange du papier. En troisième, ils doivent produire quelque chose sur le thème du labyrinthe et ils doivent se perdre. Ils font ça soit en deux dimensions, soit en trois dimensions avec une construction, ou bien une vidéo de deux minutes maximum. On a aussi fait un travail de photos sur le chemin entre chez eux et le collège. » (Journal du 7 mai 2009)

De la même façon, une professeure de musique landaise arrivant en milieu de carrière a fait télécharger à ses élèves de troisième SEGPA un extrait du film Le masque de Zorro sorti en 1998. Dans cet extrait, Zorro mène un duel à l'épée avec une femme. Ce duel va se transformer en danse : les bruits des bottes des duellistes vont peu à peu se rythmer et devenir à la fois une danse et une musique. L'enseignante essaye de faire que les élèves perçoivent la musique comme un élément signifiant du film. Les élèves ont leur ordinateur portable allumé et la professeure a branché le sien sur un vidéoprojecteur. Dans un premier temps, les élèves doivent lire un polycopié puis répondre aux questions en visionnant le film. Puis la professeure corrige en interrogeant les élèves. Dans les premiers instants de l'extrait, elle cherche à leur faire décrire les éléments musicaux qui mettent en valeur la tension dramatique de la scène. Différencier les instru- 
ments, définir le caractère de la mélodie, repérer l'aspect décousu et fragmentaire de l'orchestration à cet instant et saisir que la musique crée une ambiance de " suspense ». Ensuite vient une scène de duel dans laquelle, les bruits de bottes des duellistes vont se rythmer et le duel va devenir danse. La musique accompagne cette évolution :

- la professeure : Qu'est-ce qu'on pourrait faire sur cette musique?

- Danser.

- p : Voilà c'est dansant. Est-ce qu'elle est bien adaptée à la scène ? C'est une scène de quoi?

- De combat.

- p : Alors ?

- En même temps c'est comme s'ils dansaient.

- C'est un combat avec de la danse.

- $\mathrm{p}$ : Mais alors pourquoi on a ce genre de musique avec un combat?

- Parce que c'est pas vraiment un combat.

- p : Voilà, et donc la musique nous dit que c'est un combat qui ne sera pas dramatique : il n'y aura pas de morts. Dans ce qui vient, dites-moi ce qui se passe au niveau des pieds.

- Cela fait un rythme.

- Un rythme qui va bien avec la musique.

- p : Voilà le bruit devient percussion et devient musique. (Journal du 16 décembre 2004, cf. Khaneboubi, 2007)

Les enseignants de disciplines pour lesquels les enjeux sont plus importants ont des utilisations moins risquées. Une enseignante de mathématiques décrivait son utilisation de cette façon :

"J'utilise SMA0, Géoplan, et des logiciels d'entraînement en salle info une séance de temps en temps parfois en classe entière, parfois en demi-groupe. Je n'ai que des classes de sixième et cinquième. Je fais comme ça parce qu'il y une évaluation dans ces logiciels-là. Je ne vois pas l'intérêt s'il n'y a pas d'évaluation. Maintenant qu'ils ont un ordi je les réoriente vers Mathadoc et Mathenpoche. » (Journal du 25 mai 2009) 
Cette enseignante de physique présente elle aussi une utilisation peu risquée :

"Je fais tous mes cours sur diaporama. Pratiquement tous les cours, toutes les classes. Lorsque l'on fait des séances en salle informatique c'est surtout pour faire des recherches sur internet par exemple la dernière fois ils ont fait des recherches sur l'histoire de l'atome. Sinon j'ai obtenu le livre numérique par notre éditeur ce qui fait que je le projette avec le vidéo projecteur et les élèves n'ont pas besoin d'apporter leur livre. » (Journal du 11 juin 2009)

Les utilisations les plus répandues des ordinateurs portables sont souvent assez banales (Rinaudo, 2008). Daguet (2007) estime, dans les Landes, que « même si [les] enseignant[s] intégrai[ent] de plus en plus les TIC dans les phases de préparation de cours (sujets d'examens, polycopiés, création de ressources...), les usages informatiques lors du face à face pédagogique restaient proportionnellement moins importants. »

Souvent, les enseignants qui utilisent les TIC fréquemment le font dans la continuité d'utilisations de technologies antérieures comme le magnétophone, le magnétoscope, le rétroprojecteur, etc. Par exemple, un professeur d'anglais isarien présentait son utilisation du TBI ainsi : «On peut faire la même chose avec le tableau ordinaire, mais le TBI c'est plus sympa» (Journal du 7 mai 2009). En somme, les apports spécifiques paraissent modestes. Au travers des observations et des entretiens, on note que les enseignants qui utilisent les ordinateurs pour faire la classe font souvent remarquer qu'ils pourraient conduire leurs cours de la même façon avec des technologies plus anciennes mais qu'avec les ordinateurs ils disposent d'une plus grande facilité de manipulation des documents en cours ce qui leur permet de focaliser leur attention sur les interactions avec les élèves. L'extrait de journal décrivant un cours de musique ci-dessus constitue une illustration de cette idée.

On explique souvent les non-usages et les mésusages par un manque de formation des enseignants. Pourtant, dans les Landes et dans l'Oise, les deux enquêtes par questionnaires montrent que les professeurs utilisant le plus souvent l'ordinateur en classe sont d'une part ceux qui ne sont pas les mieux dotés en savoir-faire techniques et, d'autre part, ce ne sont pas les plus jeunes, mais plutôt ceux qui ont une expérience d'enseignement conséquente (Khaneboubi, 2009).

\section{Discussion et perspectives}

Pour les enseignants ces projets ne constituent pas une rupture importante dans leurs réalisations quotidiennes. Nous n'avons pas constaté une grande diversité dans les utilisations en classe, excepté en musique et en art plastique. 
Néanmoins, cet élément mériterait d'être examiné avec davantage de précisions. Il serait fructueux de mener une nouvelle recherche d'inspiration didactique avec des enseignants de musique pour chercher à établir une typologie des usages et des applications les plus utilisées sur un tronçon de curriculum.

En outre, la formation technique des enseignants est un élément qui revient dans les discours des enseignants des Landes et de l'Oise. Ils estiment que l'implication des rectorats reste toute relative et peine à répondre à la demande de formation émanant des équipes pédagogiques. Il serait intéressant d'analyser dans quelle mesure les dispositifs de formation continue portant sur les TIC s'adaptent aux situations de saturation technologique en classe.

On peut aussi s'interroger sur les apports pour les élèves de ce type de dotation. Leurs usages sont le plus souvent des pratiques d'adolescents plus que de collégiens (Rinaudo, 2008). Cela a été bien décrit par Rinaudo et ses collègues (2008) dans leur rapport qu'ils ont rédigé pour le conseil général d'Ille-et-Vilaine sur le projet Ordi 35 :

"On peut donc remarquer, sans que cela soit particulier au dispositif Ordi 35, que les jeunes qui utilisent les messageries pour échanger certes fréquentent leurs camarades, mais, paradoxalement, restent au domicile familial, dans un univers plus sûr, mais avec une autonomie moindre qu'en sortie. C'est en fait le temps passé avec les pairs qui s'étire et envahit, via les technologies de communication, l'univers familial. [...] En fait, on pourrait avancer que les collégiens, à l'âge de l'adolescence, se saisissent d'Ordi 35 comme d'un outil qui permet tout à la fois le lien vers l'extérieur, mais aussi le lien vers la famille, ce qui n'est pas sans évoquer les propriétés de l'espace transitionnel formalisées par Winnicott. $\gg$

Du point de vue des foyers ces opérations de déploiement ont certainement un impact plus important qu'en contexte scolaire. Elles présentent d'autres perspectives de recherches qui sont probablement prometteuses d'un point de vue scientifique. Les utilisations du web et des instruments informatisés soulèvent des interrogations inédites sur les processus d'organisation des communautés virtuelles ainsi que sur les productions et les créations de tout à chacun.

Enfin, il est utile d'insister sur la particularité des dispositifs de dotation qui ont été décrits ici de façon générale. Les dispositifs français ne sont pas mis en œuvre par les rectorats, mais par des collectivités territoriales n'ayant pas autorité sur les contenus et sur les modes d'enseignements, mais uniquement sur le matériel. Pour aller plus loin et mieux saisir les apports de ces dispositifs, il fau- 
drait se placer dans une perspective historique et internationale et ainsi comparer les effets de politiques différentes dans des contextes différents.

\section{Conclusion}

Des dispositifs de dotation des élèves et des enseignants en ordinateurs portables ont été mis en place dans cinq départements. Ces collectivités territoriales ont manifesté la volonté de réduire la fracture numérique au travers d'opérations de communication conséquentes. Cela s'inscrit le plus souvent dans une continuité des politiques éducatives de ces institutions. Mais les curricula et les instructions émanent du ministère de l'Éducation nationale et sont mis en place par les rectorats. Il est possible que l'évolution de la situation révèle des processus d'ajustements nouveaux intéressant les recherches en éducation.

Tous les élèves et tous les enseignants reçoivent un ordinateur portable pour une année au moins, sur lesquels ils disposent des droits d'administrateur. Les collèges sont aussi équipés en matériels périphériques et en TBI. Dans l'Oise, l'Ille-et-Vilaine et les Bouches-du-Rhône les élèves ont un crédit d'internet mensuel qui leur permet d'avoir accès à la maison à des ressources éducatives comme des encyclopédies, des services parascolaires ou documentaires et souvent un ENT.

Les ressources éducatives accompagnant les dotations ne sont pas les instruments vers lesquels les enseignants se tournent en premier lieu. Lorsqu'ils utilisent l'ordinateur en classe les enseignants que j'ai rencontrés, font le plus souvent appel aux suites bureautiques ordinaires ou aux logiciels reconnus comme légitimes dans leur champ disciplinaire et/ou professionnel. Peu de productions logicielles particulières sont produites par les rectorats ou les conseils généraux, si ce n'est au travers d'initiatives enseignantes localisées. Les logiciels libres restent très utilisés dans les collèges et notamment en Corrèze. Envisager une étude comparant les usages de logiciels propriétaires et libres dans des contextes scolaires similaires constitue certainement une piste de recherche fructueuse.

Je tiens à remercier ici Georges-Louis Baron et Éric Bruillard pour avoir relu, critiqué et tempéré les premières versions de ce texte.

\section{BIBLIOGRAPHIE}

(Bellocq, 2004) 
BELLOCQ G., (2004). Gabriel Bellocq Vice-Président du conseil général des Landes. Landes interactives 2004 "un collégien, un ordinateur portable» : vers un nouvel espace numérique éducatif. Actes DU COLLOQUE DE Moliets, 7 MAI 2004. Mont-de-Marsan : conseil général des Landes, p. 2-3.

\section{(Brandt-Pomares et Boilevin, 2009)}

BRANDT-POMARES P., BOILEVIN J.-M., (2009). Ordinateurs portables et médiations dans l'enseignement : le cas de deux situations en physique et en technologie. Environnements numériques en milieu scolaire: Quels usages et quelles pratiques? J.-L. Rinaudo et F. Poyet (Ed.), Lyon : INRP.

(Chaptal 2007)

CHAPTAL A. (2007). L'illusion de modernité : approches américaines. Les dossiers de l'ingénierie éducative, décembre, $\mathrm{n}^{\circ}$ 60, pp. 54-57. Disponible sur internet: http://www.cndp.fr/archivage/valid/93854/93854-15526-19514.pdf (consulté le 10 octobre 2009).

(Corrèze 2009a)

CONSEIL GÉNÉRAL DE LA CORRÈZE, (2009). Linux expliqué aux petits et aux grands. Corrèze magazine : le mensuel du conseil général de la Corrèze, ${ }^{\circ} 65$, pp. 16-17. Disponible sur internet :

http://www.cg19.fr/fileadmin/user_upload/Correze_Magazines/2009/correze_mag_65_B D.pdf (consulté le 11 octobre 2009).

(Corrèze 2009b)

CONSEIL GÉNÉRAL DE LA CORRÈZE, (2009). Les premiers ordinateurs distribués. Corrèze magazine: le mensuel du conseil général de la Corrèze, ${ }^{\circ} 66$, pp. 17 . Disponible sur internet :

http://www.cg19.fr/fileadmin/user_upload/Correze_Magazines/2009/correze_mag_66bd.pdf (consulté le 11 octobre 2009).

\section{(Corrèze 2009c)}

CONSEIL GÉNÉRAL DE LA CORRÈZE, (2009). «Le plus beau des cadeaux : votre réussite !». Corrèze magazine : le mensuel du conseil général de la Corrèze, $n^{\circ} 67, \mathrm{pp} .17$. Disponible sur internet :

http://www.cg19.fr/fileadmin/user_upload/Correze_Magazines/2009/correze_mag_67_B D.pdf (consulté le 12 décembre 2009).

(Cuban, 2001)

CUBAN L., (2001). Oversold and underused : computers in the classroom, Cambridge : Harvard University Press.

(Daguet, 2007)

DAGUET H., (2007). Un ordinateur portable pour tous. Les Cahiers Pédagogiques, $\mathrm{n}^{\circ}$ 457. Disponible sur internet: http://www.cahierspedagogiques.com/article.php3?id_article=2882 (consulté le 10 octobre 2009). 


\section{Mehdi KHANEBOUBI}

(Daguet, 2009)

DAGUET H., (2009). La mise à disposition d'ordinateurs portables et ses effets sur la pédagogie et les usages Tice des enseignants : le cas de l'opération landaise « un collégien, un ordinateur portable». Environnements numériques en milieu scolaire: Quels usages et quelles pratiques? J.-L. Rinaudo et F. Poyet (Ed.), Lyon : INRP.

(Khaneboubi, 2007)

KHANEBOUBI M., (2007). Usages de l'informatique au collège et habitus professionnels des enseignants : exemple de l'opération "un collégien, un ordinateur portable » dans le département des Landes. Thèse de doctorat, Université Bordeaux 2.

(Khaneboubi 2009)

KHANEBOUBI M., (2009). Facteurs influençant les usages de l'informatique en classe par des enseignants des collèges du département des Landes. Informatique et progiciels en éducation et en formation. G.-L. Baron, E. Bruillard et L.-O. Pochon, Lyon : ENS Cachan, IRDP et INRP.

(Larose, Grenon, Pearson, Marin et Lenoir , 2004)

LAROSE, F., GRENON, V., PEARSON, M., MARIN, J. F., \& LENOIR, Y. (2004). Les facteurs sociolgiques et pédagogiques qui affectent les pratiques des enseignants du primaire au regard de l'informatique scolaire. Dans J. Desbiens, J. F. Cardin, \& D. Martin (Éd.), Intégrer les TIC dans l'activité enseignante: quelle formation?, quels savoirs?, quelle pédagogie? (p. 5980). Laval, Quebec: Les presses de l'université de Laval.

(Liautard, 2007)

LIAUTARD D., (2007). Propos de bilan... À propos d'Ordina 13. Les dossiers de l'ingénierie éducative, décembre, $\mathrm{n}^{\circ} 60$, pp. 58-62. Disponible sur internet :

http://www.cndp.fr/archivage/valid/93855/93855-15525-19513.pdf (consulté le 10 octobre 2009)

(Rinaudo, 2008)

RINAUDO J.-L., (2008). Ordinateurs portables et construction de l'identité des collégiens. Actes du colloque Journées Communications et Apprentissage Instrumentés en Réseau, p. 207-219.

(Rinaudo et al. 2008)

RINAUDO J.-L., TURBAN J.-M., DELALANDE P., OHANA D., (2008). Des ordinateurs portables, des collégiens, des professeurs, des parents : rapport de recherche sur le dispositif Ordi 35 2005-2007. Disponible sur internet :

http://www.marsouin.org/article.php3?id_article=241 (consulté le 10 octobre 2009).

\section{Webographie}

(landesinteractives.net, 2009)

http://www.landesinteractives.net (consulté le 11 octobre 2009)

(landesinteractives.net, 2010) 
http://www.landesinteractives.net/pagesEditos.asp?IDPAGE=185\&sX Menu selectedID=1 eft 1 (consulté le 4 mars 2010)

(oise.fr, 2009)

http://www.oise.fr/Ordi_60.1589.0.html (consulté le 11 octobre 2009)

(ordi35.org, 2009)

http://www.ordi35.org/index.php?id=31 (consulté le 12 décembre 2009)

(ordina13.com, 2009)

http://www.ordina13.com (consulté le 11 octobre 2009)

(peo60.fr, 2010)

http://www.peo60.fr/index.php?id=540 (consulté le 4 mars 2010)

${ }^{\mathrm{i}}$ Entretien avec Pierre Louis Ghavam responsable des technologies de l'information et de la communication au conseil général des Landes, www.landes.org (non disponible, consulté le 2 mai 2002). 